\title{
Acceptance and Commitment Therapy: A Transdiagnostic Behavioral Intervention for Mental Health and Medical Conditions
}

\author{
Lilian Dindo $^{1}$ • Julia R. Van Liew ${ }^{2}$ Joanna J. Arch ${ }^{3,4}$ \\ Published online: 7 March 2017 \\ (C) The American Society for Experimental NeuroTherapeutics, Inc. (outside the U.S.) 2017
}

\begin{abstract}
Psychological interventions have a long history of successful treatment of patients suffering from mental health and certain medical conditions. At the same time, psychotherapy research has revealed key areas of growth for optimizing patient care. These include identifying novel treatment delivery methods that increase treatment adherence, developing new strategies to more effectively address the ever-growing population of patients with comorbid conditions, and elucidating the mechanisms by which effective treatments work in order to further refine their design. Acceptance and commitment therapy (ACT) is an empirically supported psychotherapy that offers promise for patients suffering from a wide range of mental and physical conditions, while addressing these gaps and challenges in the field. ACT rests on the fundamental premise that pain, grief, disappointment, illness, and anxiety are inevitable features of human life, with the therapeutic goal of helping individuals productively adapt to these types of challenges by developing greater psychological flexibility rather than engaging in counterproductive attempts to eliminate or suppress undesirable experiences. This is achieved through committed pursuit of valued life areas and directions, even in the face of the natural desire to escape or avoid painful
\end{abstract}

Lilian Dindo

lilian.dindo@bcm.edu

1 Menninger Department of Psychiatry and Behavioral Sciences, Baylor College of Medicine and the VA HSR\&D Center for Innovations in Quality, Effectiveness and Safety, (MEDVAMC 152), 2002 Holcombe Blvd., Houston, TX 77030, USA

2 VA Central Iowa Health Care System, Des Moines, IA, USA

3 Department of Psychology and Neuroscience, University of Colorado Boulder, Boulder, CO, USA

4 Division of Cancer Prevention and Control, University of Colorado Cancer Center, Aurora, CO, USA and troubling experiences, emotions, and thoughts. ACT is transdiagnostic (applies to more than one condition), process-focused, and flexibly delivered. In a relatively short period of time, ACT has been effectively implemented across a broad range of therapeutic settings, including mental health, primary care, and specialty medical clinics. ACT has also been delivered in a variety of formats, including 1-day group workshops, online and smartphone applications, and telehealth. Focus on how best to package and deliver treatment to meet the unique needs of different patient populations helps to ensure treatment adherence and has fostered successful application of ACT for patients in everyday clinical settings.

Keywords Acceptance and Commitment Therapy · Psychotherapies $\cdot$ Mental health

\section{Introduction}

Psychological interventions have a long history of success in management of both mental illness and chronic medical symptoms and conditions, including depression, migraine headache, chronic pain, and inflammatory bowel disease $[1,2]$. Historically, treatments within the cognitive behavioral therapy domain have shown the strongest empirical support for a wide range of psychiatric and medical conditions [3]. However, psychotherapy research has also revealed important gaps and areas for further growth $[4,5]$. For example, a meta-analysis of 125 studies of this traditional form of outpatient psychotherapy found that $50 \%$ of patients terminate their participation prematurely, with nearly $40 \%$ dropping out after their first or second visit [6]. Thus, the traditional delivery model of weekly individual psychotherapy for several months does not reflect the preferences or utilization practices of most patients [7]. As treatment adherence and completion are often the greatest obstacle 
to effective mental health care, there is a tremendous unmet need for development of novel delivery methods that improve treatment adherence. In addition, it is also important to identify the key mechanisms and components of therapy that lead to adaptive change, in order to optimally refine and emphasize effective components while eliminating nonactive or even counterproductive ingredients $[8,9]$. At the same time, the importance of psychological interventions that better meet the needs of patients with comorbid psychiatric and medical conditions through a unified, transdiagnostic approach has also become increasingly recognized [10]. Furthermore, novel psychotherapy treatment delivery models adapted to the preferences and utilization practices of patients in everyday clinical settings, such as brief treatment courses and integrated service delivery, are currently desired.

The need for interventions that meet the above challenges is particularly great given the rapidly growing comorbidity challenge facing the healthcare community. For example, by 2030 an estimated 171 million Americans will be living with multiple chronic medical conditions [11], with a predicted $10 \%$ to $20 \%$ having co-occurring major depressive or anxiety disorders [12-14]. Comorbidity of medical and psychiatric illnesses adversely affects symptom burden, quality of life, overall function, and prognosis $[13,15,16]$, and is also associated with higher healthcare expenses through increased healthcare complexity and service utilization. Sadly, this results in overall lower-quality healthcare $[17,18]$. Furthermore, the influence of mental health and medical conditions is bidirectional, further magnifying the challenges faced by patients and healthcare providers. For example, diagnosis and treatment of medical illnesses, as well as their accompanying dysfunction or disability, commonly induce strong emotional distress [12]. In addition, adhering to treatment recommendations and engaging in illness self-management are even more challenging for patients with comorbid mental health conditions that interfere with motivation and drive [14]. Patients with depression, for example, are three times less likely than nondepressed patients to adhere to treatment recommendations [19]. Additionally, the way in which individuals cope with illness and the associated stress appears to have important long-term effects [20]. For example, avoidant cognitive and behavioral coping strategies, such as ignoring medical reminders or using distraction, mental disengagement, and denial [21], are associated with poorer psychological and health outcomes [22,23]. Conversely, interventions directed at countering ineffective avoidance strategies and encouraging engagement in important activities improve health outcomes [24-26].

Thus, it is evident that meeting today's mental health needs requires a transdiagnostic, flexibly delivered psychotherapeutic approach that can be readily integrated in diverse healthcare settings, and which focuses on identification and further refinement of adaptive coping strategies. Acceptance and commitment therapy (ACT, pronounced as one word, as in "to act" [27, 28]) represents a behavioral intervention model with the capacity to address these multiple requirements.

\section{ACT}

Over the past 25 years, behavioral interventions that incorporate mindfulness and acceptance have emerged as an evolution of the cognitive behavioral psychotherapy tradition [29]. One of these novel modalities is ACT, which begins with the fundamental understanding that pain, grief, loss, disappointment, illness, fear, and anxiety are inevitable features of human life. The goal of ACT is not elimination or suppression of these experiences. Rather, ACT emphasizes pursuit of valued life areas and directions, such as intimate relationships, meaningful work, and personal growth, in the face of these painful experiences [28]. Increased engagement in meaningful life activities, even while experiencing negative thoughts and emotions or other difficulties, is accomplished in ACT by cultivating psychological flexibility (see below).

In the original ACT model, experiential avoidance - the unwillingness to remain in contact with uncomfortable private events (e.g., thoughts, feelings, and physiological sensations) by escaping or avoiding these experiences [27] in ways that have (long-term) negative consequences-was posited as the central difficulty associated with distress and dysfunction. Avoidance reduces immediate contact with distressing experiences and thus provides short-term relief (e.g., drinking alcohol to reduce anxiety). In the long term, however, avoidance leads to greater dysfunction and increased distress. The willingness to experience or "make room for" difficult internal events in pursuit of one's values and goals was seen as an antidote to experiential avoidance. More recently, the term psychological flexibility has been used to describe the proposed key therapeutic processes in the ACT model. Psychological flexibility, defined as the ability to persist in or change behavior when it is in service of valued ends in a particular context, reflects the broader target of the ACT approach [28].

Psychological flexibility is cultivated in ACT by strengthening the following six core skills: 1 ) flexibly and purposefully remaining in the present moment by being mindful of thoughts, feelings, bodily sensations, and action potentials, including during distressing experiences ( $v s$ losing contact with the present); 2) keeping balanced and broad perspective on thinking and feeling, such that painful or distressing thoughts and feelings do not automatically trigger maladaptive avoidance behaviors (vs poor perspective taking skills); 3 ) clarifying fundamental hopes, values, and goals such as being there for one's family, pursuing meaningful work, and so on ( $v s$ being disconnected from the things and people that matter most); 4) cultivating commitment to doing things in line with identified hopes, values, and goals ( $v s$ failing to take needed behavioral steps in accord with core values); 5) willingly 
accepting the unwanted feelings inevitably elicited by taking difficult actions, particularly those consistent with patients' hopes, values, and goals (vs making efforts to control or eliminate difficult internal experiences); and 6) defusion, or stepping back from thoughts that interfere with valued actions and seeing them for what they are ( $v s$ seeing thoughts as literal truths). Key in the ACT model is also "workability"-helping develop greater awareness of one's behaviors and whether those behaviors are "working" in terms of effectively solving the problem and of moving one toward valued ends. For example, in the case of anxiety, a patient may be asked if his/her use of alcohol (or any other avoidance strategy) is working in the long run in terms of solving the problem of anxiety; and examining the costs of using this strategy in the long run on valued life areas. Importantly, nonjudgmental identification of "unworkable" behavioral patterns motivated by short-term symptom relief helps to facilitate behavior changes that are aligned with long-term valued ends [27, 28].

\section{ACT is transdiagnostic}

Rather than address a specific symptom or disorder with an outcome-focused goal of symptom reduction, ACT was developed to promote greater flexibility with regard to the set of 6 interrelated and overarching psychological processes outlined above. Each of these processes are psychological skills that can be enhanced in any domain of life with regard to unwanted internal experiences or symptoms (e.g., thoughts, feelings, physical sensations). Thus, ACT has broad applicability, goes beyond any single mental or physical health condition, and offers a unified model of behavioral change with multiple favorable outcomes. These include helping people cope with psychological difficulties ranging from psychosis to depression, facilitating behavioral change associated with improving medical conditions (e.g., health behavior change or illness self-management), simultaneously addressing comorbid medical and psychiatric conditions, and even mitigating subsyndromal concerns. The primary goal of ACT in any patient population is to foster greater psychological flexibility in the face of challenges while optimizing active engagement in one's own life. The end result is greater life satisfaction in the face of a wide variety of mental and physical challenges.

Of note, although ACT interventions do not focus directly on symptom change, research has shown that symptom reduction is a by-product of re-engaging in life in meaningful ways and increasing acceptance of difficult internal experiences [9, 30]. For example, as specifically applied to treating anxiety disorders and related symptoms, the ACT model proposes that it is the preoccupation with and struggle against anxiety that is the primary cause of disability and suffering, rather than the presence of anxiety per se [31]. Patients are encouraged to examine the cost of "fighting against anxiety", which commonly includes maladaptive behaviors, such as avoiding numerous meaningful activities, forcing a family member to accompany one everywhere, and not pursuing one's full career or relationship ambitions. The ACT therapist would teach the patient new ways of being with anxiety, such as simply noticing it for what it is (a cluster of thoughts, feelings, and physical sensations), opening up to experiencing anxiety as it is (as an ongoing stream of shifting thoughts, feelings and sensations), letting go of efforts to control or avoid experiencing anxiety (and thus reducing the high cost of such efforts), and learning to re-engage in avoided yet personally valued activities, even in the presence of unwanted anxiety. The focus on pursuing valued behavior and changing one's relationship to anxiety, from struggle to acceptance, has the paradoxical effect of reducing anxiety disorder symptoms, to the same extent or more than traditional, cognitive behavioral approaches, as suggested by large randomized clinical trials (RCTs) of ACT for anxiety disorders [32-35] (for a review, see Landy et al. [36]).

In another example, as applied to chronic pain conditions, patients are taught to notice the differences between the physical sensations of pain and their reactions to them, as well as the effect that these reactions have on their mental health and functioning. This is because patients' cognitive and emotional reactions to pain may exacerbate the intensity of their painful experience. Patients may get frustrated and have thoughts such as, "Why me? I don't have time for this. I can ${ }^{1} t$ stand this. This is awful. I cannot go out because something may happen". ACT teaches patients more adaptive responses, such as, "I am experiencing throbbing pain. It feels warm. I have experienced this before. I will go for a walk with my friend and check in to see how I feel afterwards". Both situations involve pain; the former may exacerbate the pain, while the latter acknowledges the pain without exacerbating it. In addition to exacerbating the pain, viewing pain as "unacceptable" may lead to maladaptive avoidance of situations that may trigger pain, and can also lead to increased pain pill taking, which often carries significant costs to quality of life, increased depression, and possible addiction. Thus, patients are invited to examine the "workability" of their pain avoidance strategies (i.e., whether they actually help reduce pain in the long run), as well as to recognize the effects of avoidance on life engagement and vitality. Mindful, nonjudgmental observation of the limited effectiveness of current avoidance strategies facilitates re-engagement in life in meaningful and valuable ways. Research has consistently shown that an ACT-based approach to chronic pain leads to improved functioning and quality of life [24, 37-40].

\section{ACT Meets Implementation Needs}

\section{ACT is Flexibly Delivered}

Importantly, ACT has been effectively implemented in various treatment-delivery formats and settings [41]. Flexibility in 
delivery format and duration allows focus to be placed on how best to package and deliver the intervention to meet the needs of the patient population, ensure treatment adherence, and increase successful dissemination into everyday clinical settings. For example, one ACT delivery model being used is 1-day (4$6 \mathrm{~h}$ ) ACT group workshops. These workshops have been successfully provided to patients with a range of medical conditions, including diabetes, multiple sclerosis, obesity, and surgery, as well as patients with comorbid medical and psychiatric conditions such as migraine and depression, inflammatory bowel disease and depression, and cardiovascular disease and anxiety (e.g., Gregg et al. [42], Sheppard et al. [43], Lillis et al. [44] and Dindo [45]). Outcomes include improvements in glycated hemoglobin levels (in diabetes), increased quality of life, decreased subjective sense of distress, and improved disease management.

Interestingly, presenting the treatment as a "workshop" rather than "therapy" has several advantages. To begin, this terminology is better suited for patients for whom seeking mental health care is associated with stigma [46, 47]. Also, the 1-day format ensures treatment adherence and completion because it is more accessible and feasible than weekly treatments, particularly for patients who live in rural communities or suffer other functional barriers to accessing care [48]. Finally, this more concentrated and time-limited approach is better suited for primary care settings in which patients may have different expectations from those explicitly seeking mental health care [49]. For example, medical patients often do not spontaneously report distress, psychiatric symptoms, or functional impairment to their healthcare providers, and also may not recognize the negative impact that mental distress has on their health [50]. Although this literature is still relatively young, the positive results obtained with 1-day workshops challenge the assumption that therapy needs to be provided in the traditional weekly session format [45]. Also, providing a 1-day (4-6 h) workshop still offers patients more treatment or contact time than is commonly obtained in community settings. As noted above, the lack of treatment adherence and completion is often the greatest obstacle to effective delivery of psychotherapy services [6].

\section{ACT can be Integrated Into Medical Care Settings and Populations}

Embedding behavioral interventions within medical settings provides broad access and more comprehensive care for the behavioral aspects of medical conditions and mental health problems. Such systems-based approaches can result in improved overall outcomes in mental health and other medical conditions. Robinson and Strosahl [51] pioneered the successful integration of brief behavioral interventions into primary care settings, with flexible use of ACT content across numerous models and studies. In the context of specialized medical care settings, growing attention has recently focused on integrating ACT-based interventions into cancer care. In the largest such trial to date [52], Australian researchers randomized 410 colorectal cancer survivors to usual care or a series of brief telephone coaching sessions focusing on leveraging ACT strategies to promote health behavior change related to preventing cancer recurrence (weight loss, diet, exercise, alcohol, smoking). At 12-month follow-up, relative to usual care, ACT led to significant improvements in physical activity, dietary habits, and body mass index. Work by Bricker et al. [53-56] has demonstrated that ACT interventions in various delivery formats (face-to-face, online, smartphone app, telephone) have led to greater smoking cessation rates than gold-standard cognitive behavioral therapy comparison interventions. Finally, building on promising pilot findings [57], ongoing work by Arch and Mitchell is currently comparing an ACT-based group intervention to usual care for cancer survivors with elevated anxiety. Importantly, this work recruits patients directly from cancer care settings and trains on-site oncology clinical social workers to deliver the intervention, thus integrating ACT into a specialty medical care setting.

\section{ACT Interventions can be Brief}

Numerous studies outlined above share a common feature: they employ brief ACT interventions. ACT has been administered successfully in 1-day workshops [45], telehealth coaching calls $[52,55]$, online and smartphone interventions [53], and 20-min sessions integrated into primary care visits [51], demonstrating the feasibility, acceptability, and preliminary efficacy of brief ACT interventions. In one study with chronic pain patients, those randomized to a 4-h ACT intervention had taken fewer sick days than the treatment-as-usual group (0.5 vs 56.0) at 6-month follow-up [58]. In a study with hospitalized psychotic patients, a 4-session ACT intervention was shown to reduce hospitalizations over a 4-month follow-up period [59].

Though we lack sufficient space to provide an exhaustive list of the literature of brief ACT interventions or ACT interventions in healthcare settings or populations, we aim to provide a sense of the breadth of this evolving literature. Though promising, numerous studies are in their pilot phases and require replication in larger samples.

\section{Empirical Support for Acceptance and Commitment Therapy}

Currently, ACT has been identified by the American Psychological Association as an empirically supported treatment for depression, mixed anxiety disorders, psychosis, chronic pain, and obsessive-compulsive disorder. It has also been endorsed by the Department of Veterans Affairs as an 
evidence-based therapy for mood disorders. For a summary of the 8 ACT meta-analyses published to date, which include interventions addressing mental or physical health symptoms, see Dimidjian et al. [9]. Briefly, several meta-analyses have shown that ACT is effective for treating patients with a wide variety of problems, with generally medium effect sizes compared with treatment as usual, but with large effect sizes compared with wait-list control. Variability exists across meta-analyses with regard to the magnitude and significance of effect sizes for ACT versus established interventions such as cognitive behavioral therapy [9]. Preliminary findings from moderation analyses further suggest that ACT may be particularly well suited to individuals suffering from comorbid medical and psychiatric conditions [60]. It is possible that the common mechanisms of change targeted in ACT function in parallel for treating individuals with a variety of psychological conditions and major stressors, including medical conditions.

The variability across meta-analyses may reflect the heterogeneity of problems addressed by the ACT literature. As of August 2016, there were 136 published RCTs of ACT in a broad range of clients, including those with mental health (e.g., depression, anxiety, psychosis, substance abuse, eating disorders), medical (e.g., chronic pain, cancer, epilepsy, diabetes), behavioral (e.g., smoking, weight loss), and other issues (e.g., stigma, parenting, chess performance, work performance, human prejudice). It is a relatively young literature base, with the earliest meta-analysis published in 2006, but is growing quickly.

\section{Core Therapeutic Processes Targeted in ACT: Experiential Avoidance/Psychological Inflexibility}

It is becoming increasingly necessary to examine not only whether treatments work, but also how they work. Understanding the specific mechanisms or processes that mediate clinical improvement in psychiatric symptoms, functional status, and/or quality of life allows for intervention optimization by refining and emphasizing the components responsible for change, and also eliminating nonactive ingredients [61]. Fundamental to the scientific basis of ACT is an emphasis on elucidating the processes that predict human behavior and examining whether influencing those processes leads to changes.

In the ACT model, psychological inflexibility is posited to underlie diverse problems, including mental health, behavioral, and comorbid complications; and there has been substantial research examining the role of psychological inflexibility in difficulties across patient populations [62,63]. Thus, ACT interventions aim to foster increased psychological flexibility (i.e., greater ability to persist in or change behavior when in service of valued ends in a particular context) by cultivating the 6 core ACT processes described above. Measures of psychological flexibility (e.g., Bond et al. [64]) or individual ACT processes (e.g., acceptance, values) have been used in many ACT clinical trials to assess the mediating role of psychological flexibility on outcomes of interest [65]. These studies have shown that psychological inflexibility and its various components relate to and predict an array of important negative outcomes. For example, cross-sectional studies in adults and young people have found positive associations between psychological inflexibility and most forms of psychopathology (e.g., Chawla and Ostafin [66]; Greco et al. [67]; Howe-Martin et al. [68]; Venta et al. [69]). Moreover, prospective studies suggest that psychological inflexibility is implicated in the onset of mood and anxiety disorders. For example, in an adult sample spanning 6 years, psychological inflexibility was associated with current mood and anxiety disorders, and also predicted changes in diagnostic status time [70]. Psychological inflexibility (and/or its components such as behavioral avoidance) also relates to negative physical health outcomes such as greater pain intensity and pain-related disability (e.g., McCracken [71]), decreased health-related quality of life and productivity, greater severity and frequency of physical symptoms, and greater healthcare utilization $[58,72,73]$.

Furthermore, ACT treatment studies have shown that psychological flexibility can be enhanced and lead to changes in important outcomes (see Hayes et al. [64] for a summary). Again, the work is too broad to describe in detail here and only a few highlights will be mentioned.

For example, in an ACT-based treatment study of patients with borderline personality disorder, improvements in psychological flexibility were found to predict improvements in depressive symptoms [74]. In a RCT for patients with diabetes, a 1-day ACT plus diabetes management intervention resulted in improved blood glucose levels, better diabetes self-care, and higher levels of diabetes-related acceptance 3 months later versus diabetes management alone. Of note, improvements in psychological flexibility mediated the relationship between group assignment and blood glucose [42]. In a RCT for a group of distressed patients at risk for vascular disease, a 1-day ACT intervention led to significant improvements in depression and anxiety. Furthermore, improvements in psychological flexibility mediated the benefits of the ACT intervention on depression and anxiety [75]. The impact of changes in individual ACT processes has also been well-studied in ACT trials for chronic pain. Consistently, increases in acceptance of pain and/or values-based action have been observed to relate to improvements in anxiety, depression, and pain-related disability during treatment or at follow-up [38-40]. In one suggestive ACT trial for chronic pain [76], psychological flexibility significantly mediated the outcomes of life satisfaction and pain-related disability, whereas non-ACT-specific processes (those specified by other theories) did not. Overall, this evidence should be viewed as promising rather than definitive, as the research base is young and growing, and many studies have been relatively small. 


\section{Discussion}

The ACT approach posits that psychological human suffering is due to a lack of behavioral flexibility and effectiveness, which emerges from experiential avoidance, cognitive entanglement, difficulty with perspective taking, loss of contact with the present, and failure to take needed behavioral steps in accord with core values [28]. Powerful alternatives are available in the ACT model through the skills of acceptance, cognitive distancing, perspective-taking, mindfulness, values, and committed action. Research is increasingly showing that these same ACT processes can help us understand and change a diverse set of problems and symptoms, including the sequelae of medical illnesses. ACT is rooted in a philosophy of science that highlights the role of contextual factors in influencing behavior-a perspective that is gaining appreciation in the medical community. Indeed, a panel of comorbidity experts recently identified understanding contextual factors, such as patients' relationships, values, and goals, as a vital paradigm shift in the treatment of patients with multiple chronic conditions [77].

Medical conditions influence and are influenced by mental health symptoms. Despite the high prevalence of comorbidity, existing treatments and service delivery approaches largely reflect fragmented, disease-specific care. Integrated, transdiagnostic treatment for comorbid mental and physical health symptoms is an increasingly valued approach for improving whole-person patient health and quality of life. For example, the Agency for Healthcare Research and Quality recently identified inclusion of mental health treatment into healthcare as 1 of 3 key priorities for future research on patients with multiple chronic conditions [78].

As a transdiagnostic and flexibly delivered approach, ACT represents a promising way to meet the heterogeneous needs and treatment preferences of patients with a broad range of problems, including mental health, medical, behavioral, and co-occurring mental and physical health symptoms. A rapidly evolving literature supports the preliminary feasibility, acceptability, and efficacy of brief ACT treatments, including those embedded in existing medical care settings. ACT interventions have been associated with improvements in mental health, medical, and behavioral outcomes, and overall functioning and quality of life (e.g., [79, 80]). Future research should assess the cost-effectiveness of such integrated approaches, which are anticipated to promote improved symptomatic and functional outcomes among comorbid patients at lower costs.

Acknowledgments This work was funded by grant number K23MH097827 from the National Institute of Mental Health, awarded to Lilian N Dindo; and was partially supported by the use and resources of the Houston VA HSR\&D Center for Innovations in Quality, Effectiveness and Safety (CIN13-413). This work was also funded by a grant from the American Cancer Society RSG-15-020-01 and PEP-16-055-01, awarded to Joanna J. Arch. The opinions expressed reflect those of the authors and not necessarily those of the Department of Veterans Affairs, the US government, or Baylor College of Medicine.

\section{References}

1. Barlow DH. Psychological treatments. American Psycholc 2004; 59.9: 869.

2. Seligman, MEP. The effectiveness of psychotherapy: the Consumer Reports study. Am Psychol 1995; 50:965-974.

3. Kroenke K, Swindle R. Cognitive-behavioral therapy for somatization and symptom syndromes: a critical review of controlled clinical trials. Psychother Psychosom 2000;69:205-215.

4. Barlow DH., Levitt JT, Bufka, LF. The dissemination of empirically supported treatments: a view to the future. Behav Res Ther 1999;37: S147-S162.

5. Baker TB, McFall RM, Shoham V. Current status and future prospects of clinical psychology toward a scientifically principled approach to mental and behavioral health care. Psychol Sci Public Interest 2008;9(2):67-103.

6. Wierzbicki M, Pekanik G. A meta-analysis of psychotherapy dropout. Prof Psychol: Res Pr 1993;24:190-195.

7. Wierzbicki M, Pekanik G. The relationship between expected and actual psychotherapy treatment duration. Psychotherapy 1986;23: 532-534.

8. Kazdin A.E Mediators and mechanisms of change in psychotherapy research. Ann Rev Clin Psychol 2007; 3:1-27.

9. Dimidjian S, Arch JJ, Schneider RL, Desormeau P, Felder JN, Segal ZV. Considering meta-analysis, meaning, and metaphor: A systematic review and critical examination of "third wave" cognitive and behavioral therapies. Behav Ther 2016;47:886-905.

10. Barlow DH, Allen LB, Choate ML Toward a unified treatment for emotional disorders. Behav Ther 2004;35(2):205-230.

11. Robert Wood Johnson Foundation. Chronic Care: Making the Case For Ongoing Care. Princeton, NJ: Robert Wood Johnson Foundation; 2010.

12. Evans DL, Charney DS, Lewis L et al. Mood disorders in the medically ill: scientific review and recommendations. Biol Psychiatry 2005;58:175-189.

13. Lowe B, Spitzer RL, Williams JB et al. Depression, anxiety and somatization in primary care: syndrome overlap and functional impairment. Gen Hosp Psychiatry 2008;30:191-199.

14. Merikangas KR, Ames M, Cui L, et al. The impact of comorbidity of mental and physical conditions on role disability in the US adult household population. Arch Gen Psychiatry 2007;64(10):1180-1188.

15. Kroenke K, Spitzer RL, Williams JB, et al. Anxiety disorders in primary care: prevalence, impairment, comorbidity, and detection. Ann Intern Med 2007;146:317-325.

16. Mitchell AJ, Vaze A, Rao S. Clinical diagnosis of depression in primary care: a meta-analysis. Lancet 2009;374:609-619.

17. Lurie IZ, Manheim LM, Dunlop DD. Differences in medical care expenditures for adults with depression compared to adults with major chronic conditions. J Ment Health Policy Econ 2009;12:87-95.

18. Domino ME, Beadles CA, Lichstein JC, et al. Heterogeneity in the quality of care for patients with multiple chronic conditions by psychiatric comorbidity. Med Care 2014;52(3 Suppl. 2):S101-S109.

19. DiMatteo MR, Lepper HS, Croghan TW. Depression is a risk factor for noncompliance with medical treatment: meta-analysis of the effects of anxiety and depression on patient adherence. Arch Intern Med 2000;160:2101-2107.

20. Spleen AM, Lengerich EJ, Camacho FT, Vanderpool RC. Health care avoidance among rural populations: results from a nationally representative survey. J Rural Health 2014; 30:79-88.

21. Moos RH, Schaefer JA. Coping resources and processes: current concepts and measures. In: Goldberger L, Breznitz S (eds). Handbook of Stress: Theoretical and Clinical Aspects. 2nd. New York City: Free Press; 1993. pp. 234-257. 
22. Carver CS, Pozo C, Harris SD, et al. How coping mediates the effect of optimism on distress: a study of women with early stage breast cancer. J Pers Soc Psychol 1993;65:375-390.

23. Friedman LC, Nelson DV, Baer PE, et al. Adjustment to breast cancer: a replication study. J Psych Oncol 1991;8:27-40.

24. McCracken LM, Vowles KE. Acceptance of chronic pain. Curr Pain Headache 2006; 10:90-94.

25. Vowles KE, McNeil DW, Gross RT, et al. Effects of pain acceptance and pain control strategies on physical impairment in individuals with chronic low back pain. Behav Ther 2007;38:412-425.

26. Viane I, Crombez G, Eccleston C, et al. Acceptance of the unpleasant reality of chronic pain: effects upon attention to pain and engagement with daily activities. Pain 2004;112:282-288.

27. Hayes SC, Strosahl KD, Wilson KG. Acceptance and commitment therapy: an experiential approach to behavior change. New York: Guilford Press, 1999.

28. Hayes S, Strosahl K, Wilson K. Acceptance and commitment therapy: the process and practice of mindful change. New York: Guilford Press, 2011.

29. Hayes SC, Follette VM, M. LM. Mindfulness and acceptance: expanding the cognitive-behavioral tradition. New York City: Guilford Press, 2004.

30. A-Tjak J, Davis ML, Morina N, et al. A meta-analysis of the efficacy of acceptance and commitment therapy for clinically relevant mental and physical health problems. Psychother Psychosom 2015;84:30-36

31. Forsyth J P, Eifert GH, Barrios V. Fear conditioning in an emotion regulation context: A fresh perspective on the origins of anxiety disorders. In Craske MG, Hermans D, Vansteenwegen E (eds). From basic processes to clinical implications. Washington, D.C.: American Psychological Association, 2006.

32. Arch JJ, Eifert GH, Davies C, Plumb JC, Rose RD, Craske MG. Randomized clinical trial of cognitive behavioral therapy versus acceptance and commitment therapy for the treatment of mixed anxiety disorders. J Consult Clin Psychol 2012;80(5):750-765.

33. Craske MG, Niles AN, Burklund LJ, et al. Randomized controlled trial of cognitive behavioral therapy and acceptance and commitment therapy for social anxiety disorder: outcomes and moderators. J Clin Consult Psychol 2014;82(6):1034-1048.

34. Roemer L, Orsillo SM, Salters-Pedneault K. Efficacy of an acceptance-based behavior therapy for generalized anxiety disorder: Evaluation in a randomized controlled trial. J Consult Clin Psychol 2008;76(7):1083-1089.

35. Hayes-Skelton SA, Roemer L, Orsillo SM. A randomized clinical trial comparing an acceptance-based behavior therapy to applied relaxation for generalized anxiety disorder. J Consult Clin Psychol 2013;33(8):965-978

36. Landy LN, Schneider RL, Arch JJ. Acceptance and commitment therapy for the treatment of anxiety disorders: a concise review. Curr Opin Psychol 2014;2:70-74.

37. McCracken LM, Vowles KE, Eccleston C. Acceptance-based treatment for persons with complex, long standing chronic pain: a preliminary analysis of treatment outcome in comparison to a waiting phase. Behav Res Ther 2005;43(10):1335-1346.

38. Vowles KE, McCracken LM. Acceptance and values-based action in chronic pain: a study of treatment effectiveness and process. J Consult Clin Psychol 2008;76(3):397-407.

39. Vowles KE, Wetherell JL, Sorrell JT. Targeting acceptance, mindfulness, and values-based action in chronic pain: findings of two preliminary trials of an outpatient group-based intervention. Cogn Behav Pr 2009;16(1):49-58.

40. McCracken LM, Gutierrez-Martinez O. Processes of change in psychological flexibility in an interdisciplinary group-based treatment for chronic pain based on acceptance and commitmenttherapy. Behav Res Ther 2011;49(4):267-274.
41. Ruiz FJ. A review of acceptance and commitment therapy (ACT) empirical evidence: correlational, experimental psychopathology, component, and outcome studies. Int J Psychol Psychol Ther 2010;10:125-162

42. Gregg JA, Callaghan GM, Hayes SC, Glenn-Lawson JL. Improving diabetes self-management through acceptance, mindfulness, and values: a randomized controlled trial. J Consult Clin Psychol 2007;75:336-343.

43. Sheppard SC, Forsyth JP, Hickling EJ, Bianchi J. A novel/ application of acceptance and commitment therapy for psychosocial problems Associated with multiple sclerosis. Int J MS Care 2010;12:200-206.

44. Lillis J, Hayes SC, Bunting K, Masuda A. Teaching acceptance and mindfulness to improve the lives of the obese: a preliminary test of a theoretical model. Ann Behav Med 2009; 37:58-69.

45. Dindo L. One-day acceptance and commitment training workshops in medical populations. Curr Opin Psychol 2015;2:38-42.

46. Mohr DC, Hart SL, Howard I, et al. Barriers to psychotherapy among depressed and nondepressed primary care patients. Ann Behav Med 2006:32:254-258.

47. Pietrzak RH, Johson DC, Goldstein MB, et al. Perceived stigma and barriers to mentalhealth care utilization among OEF-OIF veterans. Psychiatr Serv 2009;60(8):1118-1122.

48. Arcury TA, Gesler WM, Preisser JS, Sherman J, Spencer J, Perin J. The effects of geography and spatial behavior on health care utilization among the residents of a rural region. Health Serv Res 2005;40(1)135-156

49. Backenstrass M, Joest K, Frank A, et al. Preferences for treatment in primary care: a comparison of nondepressive, subsyndromal and major depressive patients. Gen Hosp Psychiatry 2006;28:178-180.

50. Bell RA, Franks P, Duberstein PR, et al. Suffering in silence: reasons for not disclosing depression in primary care. Ann Fam Med 2011;9:439-446.

51. Robinson PJ, Strosahl K D. Behavioral health consultation and primary care: Lessons learned. J Clin Psychol Med Settings 2009;16(1), 58-71.

52. Hawkes AL, Chambers SK, Pakenham KI, et al. Effects of a telephone-delivered multiple health behavior change intervention (CanChange) on health and behavioral outcomes in survivors of colorectal cancer: a randomized controlled trial. J Clin Oncol 2013;31:2313-2321.

53. Bricker J, Wyszynski C, Comstock B, Heffner JL. Pilot randomized controlled trial of web-based acceptance and commitment therapy for smoking cessation. Nicotine Tob Res 2013; 15(10), 1756-1764.

54. Bricker, JB, Bush T, Zbikowski SM, Mercer LD, Heffner JL. Randomized trial of telephone-delivered acceptance and commitment therapy versus cognitive behavioral therapy for smoking cessation: a pilot study. Nicotine Tob Res 2014;16:1446-1454.

55. Bricker JB, Mull KE, Kientz JA, et al. Randomized, controlled pilot trial of a smartphone app for smoking cessation using acceptance and commitment therapy. Drug Alcohol Depend 2014;143:87-94.

56. Hernández-López M, Luciano MC, Bricker JB, Roales-Nieto JG, Montesinos F. Acceptance and commitment therapy for smoking cessation: a preliminary study of its effectiveness in comparison with cognitive behavioral therapy. Psychol Addict Behav 2009;23(4):723-730.

57. Arch JJ, Mitchell J. An acceptance and commitment therapy group intervention for cancer survivors experiencing anxiety at reentry. Psychooncology 2016; 25(5):610-615.

58. Dahl J, Wilson KG, Nilsson A. Acceptance and commitment therapy and the treatment of persons at risk for long-term disability resulting from stress and pain symptoms: A preliminary randomized trial. Behav Ther 2004;35(4):785-801

59. Bach P, Gaudiano BA, Hayes SC, Herbert JD. Acceptance and commitment therapy for psychosis: intent to treat, hospitalization outcome and mediation by believability. Psychosis. 2013;5(2):166-74. 
60. Juarascio AS, Forman EM, Herbert JD. Acceptance and commitment therapy versus cognitive therapy for the treatment of comorbid eating pathology. Behav Modif 2010;34:175-190.

61. Rosen GM, Davison GC. Psychology should list empirically supported principles of change (ESPs) and not credential trademarked therapies or other treatment packages. Behav Modif 2003;27(3):300-312.

62. Hayes SC, Luoma JB, Bond FW, et al. Acceptance and commitment therapy: model, processes and outcomes. Behav Res Ther 2006;44:1-25.

63. Kashdan TB, Rottenberg J. Psychological flexibility as a fundamental aspect of health. Clinical Psychol Rev 2010;30(7): 865-878.

64. Bond FW,Hayes SC, Baer RA, et al. Preliminary psychometric properties of the Acceptance and Action Questionnaire-II: a revised measure of psychological inflexibility and experiential avoidance. Behav Ther 2011;42(4):676-688

65. Hayes SC, Livein ME, Plumb-Vilardaga J, Villatte JL, Pistorello J. Acceptance and commitment therapy and contextual behavioral science: Examining the progress of a distinctive model of behavioral and cognitive therapy. Behavior Ther 2013;44(2):180-198.

66. Chawla N, Ostafin B. Experiential avoidance as a functional dimensional approach to psychopathology: an empirical review. J Clin Psychol 2007;63(9):871-890.

67. Greco LA, Lambert W, Baer RA. Psychological inflexibility in childhood and adolescence: development and evaluation of the Avoidance and Fusion Questionnaire for Youth. Psychol Assess 2008;20(2):93.

68. Howe-Martin LS, Murrell AR, Guarnaccia CA. Repetitive nonsuicidal self-injury as experiential avoidance among a community sample of adolescents. J Clin Psychol 2012;68(7):809-829.

69. Venta A, Sharp C, Hart J. The relation between anxiety disorder and experiential avoidance in inpatient adolescents. Psychol Assess 2012;24(1), 240.

70. Spinhoven P, Drost J, de Rooij M, van Hemert AM, Penninx BW. Is experiential avoidance a mediating, moderating, independent, overlapping, or proxy risk factor in the onset, relapse and maintenance of depressive disorders?. Cogn Ther Res 2016;40(2):150-163.

71. McCracken LM. Learning to live with the pain: acceptance of pain predicts adjustment in persons with chronic pain. Pain 1998;74(1): 21-27.

72. Butler J, Ciarrochi J. Psychological acceptance and quality of life in the elderly. Qual Life Res 2007;16(4):607-615.

73. Dindo L, Turvey C, Marchman J, Recober A, O'Hara M. Depression and disability in migraine: the role of pain-acceptance and values-based living. Int J Behav Med 2014; 22(1): 109-117.

74. Berking M, Neacsiu A, Comtois KA, Linehan MM. The impact of experiential avoidance on the reduction of depression in treatment for borderline personality disorder. Behav Res Ther 2009; 47(8):663-670.

75. Dindo LN, Marchman J, Grindes H, Fiedorowicz JG. A brief behavioral intervention targeting mental health risk factors for vascular disease: a pilot study. Psychother Psychosom 2014; 84(3): 183-185

76. Wicksell RK, Olsson GL,Hayes SC. Psychological flexibility as a mediator of improvement in Acceptance and Commitment Therapy for patients with chronic pain following whiplash. Eur J Pain 2010;14:1059-e1.

77. Bayliss EA, Bonds DE, Boyd CM, et al. Understanding the context of health for persons with multiple chronic conditions: Moving from what is the matter to what matters. Ann Fam Med 2014;12 (3):260-269.

78. LeRoy L, Bayliss E, Domino M, et al. The Agency for Healthcare Research and Quality Multiple Chronic Conditions Research Network. Med Care 2014;52:S15-S22.

79. Dindo L, Recober A, Marchman JN, et al. One-day behavioral treatment for patients with comorbid depression and migraine: a pilot study. Behav Res Ther 2012; 50:537-543.

80. Dindo L, Recober A, Marchman J, O'Hara M, Turvey C. One-Day Behavioral Intervention in Depressed Migraine Patients: Effects on Headache. Headache 2014; 54: 528-538. 